\title{
AMMONIUM IONS SUPPRESS THE AMINO ACID METABOLISM INVOLVED IN THE BIOSYNTHESIS OF PROTYLONOLIDE IN A MUTANT OF STREPTOMYCES FRADIAE ${ }^{\dagger}$
}

\author{
Satoshi Ōmura, Arihiro Taki, Kazuko Matsuda and Yoshitake Tanaka \\ The Kitasato Institute, and School of \\ Pharmaceutical Sciences, Kitasato University, \\ Minato-ku, Tokyo 108, Japan
}

(Received for publication June 4, 1984)

\begin{abstract}
Protylonolide is a lactonic precursor of tylosin aglycone, produced by a mutant of Streptomyces fradiae. It originates from $n$-butyrate, propionate and acetate units. Studies were carried out using a protylonolide-producing mutant on the correlation between protylonolide biosynthesis, regulation by $\mathrm{NH}_{4}{ }^{+}$and amino acid metabolism. Protylonolide production decreased in a defined medium containing high levels of $\mathrm{NH}_{4}{ }^{+}$, but was restored by adding lower fatty acids expected to serve as precursors of protylonolide biosynthesis. Resting cell studies demonstrated that ${ }^{14} \mathrm{C}$-labeled valine, threonine, leucine, isoleucine and alanine, but not lysine, were efficiently incorporated into protylonolide, indicating that these amino acids are metabolized to lower fatty acids. The incorporation of amino acids into protylonolide was reduced when the mutant strain was previously grown under high $\mathrm{NH}_{4}{ }^{+}$conditions. We suggest that $\mathrm{NH}_{4}{ }^{+}$suppresses the relevant amino acid metabolism, thereby reducing protylonolide formation.
\end{abstract}

Tylosin biosynthesis in Streptomyces fradiae is susceptible to inhibition by $\mathrm{NH}_{4}{ }^{+}$: It decreases when high concentrations of the ions are present in a culture medium, but increases with simultaneous declines of $\mathrm{NH}_{4}{ }^{+}$level in the presence of $\mathrm{NH}_{4}{ }^{+}$-trapping agents such as zeolite. ${ }^{1,2)}$

Protylonolide $^{4)}$ (or tylactone ${ }^{3)}$ ), a key intermediate of the biosynthesis of tylosin aglycone, possesses a 16-membered lactonic structure with the same carbon skeleton as tylosin aglycone. Its production is also subject to regulation by $\mathrm{NH}_{4}{ }^{+}{ }^{1,2)}$ Such regulation appears to determine the rate of tylosin production because aglycone formation is limiting in $S$. fradiae. ${ }^{2)}$ However, little is known about the mechanism of nitrogen catabolite regulation of the biosynthesis of a macrolide aglycone.

OMMURA et al. demonstrated that tylosin aglycone originates from $n$-butyrate, propionate and acetate units. Recently, amino acids were implicated as the source of these lower fatty acid precursors, when valine was shown to be incorporated into protylonolide after deamination and decarboxylation. ${ }^{2,8)}$ It is reasonable to assume that amino acid metabolism not only supplies building units for protylonolide biosynthesis, but is also involved in its regulation by $\mathrm{NH}_{4}{ }^{+}$. To verify this, we studied reversal of $\mathrm{NH}_{4}{ }^{+}$inhibition in growing cultures, and the effect of $\mathrm{NH}_{4}{ }^{+}$on the relationship between amino acid metabolism and short-term production of protylonolide by washed mycelia. This paper deals with the results of these studies, which suggest that $\mathrm{NH}_{4}{ }^{+}$suppresses protylonolide production because of interference with relevant amino acid metabolism.

+ Bioconversion and biosynthesis of 16-membered macrolide antibiotics. XXXII. Part XXXI of this series appears in: S. Ōmura, H. Tomoda, S. Yamamoto, M. Tsukui \& H. Tanaka: Studies on two dioxygenases involved in the synthesis of tylosin in Streptomyces fradiae. Biochim. Biophys. Acta, in press. 


\section{Materials and Methods}

\section{Microorganism}

Protylonolide-producing Streptomyces fradiae strain $261,{ }^{4)}$ derived from the tylosin producer $S$. fradiae KA-427 (C-373), was used.

\section{Method of Cultivation}

Frozen mycelia of strain 261 were inoculated into 500-ml Sakaguchi flasks containing $100 \mathrm{ml}$ of a complex seed medium, ${ }^{1)}$ and incubated at $27^{\circ} \mathrm{C}$ for three days. An aliquot $(0.3 \mathrm{ml})$ of this seed culture was transferred into $50-\mathrm{ml}$ test tubes $(2 \times 20 \mathrm{~cm})$ containing $10 \mathrm{ml}$ of chemically defined media. The defined media composed of: Soluble starch $2 \%$, glucose $0.5 \%,\left(\mathrm{NH}_{4}\right)_{2} \mathrm{SO}_{4} 0.17 \sim 0.66 \%(25 \sim 100$ mM $\left.\mathrm{NH}_{4}{ }^{+}\right), \mathrm{MgSO}_{4} \cdot 7 \mathrm{H}_{2} \mathrm{O} 0.05 \%, \mathrm{~K}_{2} \mathrm{HPO}_{4} 0.05 \sim 0.22 \%(2.9 \sim 12.9 \mathrm{~mm}), 50 \%$ sodium lactate $0.55 \%$ (v/v), $\mathrm{CaCO}_{3} 0.3 \%, \mathrm{~N}$-morpholinopropanesulfonic acid (MOPS) $2.1 \%(0.1 \mathrm{M}), 0.3 \%(\mathrm{v} / \mathrm{v})$ of trace metal solution consisting of (each at $1 \mathrm{~g}$ /liter) $\mathrm{FeSO}_{4} \cdot 7 \mathrm{H}_{2} \mathrm{O}, \mathrm{MnCl}_{2} \cdot 4 \mathrm{H}_{2} \mathrm{O}, \mathrm{ZnSO}_{4} \cdot 7 \mathrm{H}_{2} \mathrm{O}, \mathrm{CuSO}_{4} \cdot 5 \mathrm{H}_{2} \mathrm{O}$ and $\mathrm{CoCl}_{2} \cdot 2 \mathrm{H}_{2} \mathrm{O}, \mathrm{pH} 7.0$ before sterilization. Solutions of $\left(\mathrm{NH}_{4}\right)_{2} \mathrm{SO}_{4}$ and MOPS in water were adjusted to $\mathrm{pH} 7.0$, separately sterilized by filtration through a Toyo-Roshi membrane filter $(0.45 \mu \mathrm{m}$, TM-2), were aseptically added before inoculation. The other conditions were as described previously. ${ }^{1,2)}$ The defined medium containing $25 \mathrm{mM} \mathrm{NH}_{4}{ }^{+}, 2.9 \mathrm{mM} \mathrm{K}_{2} \mathrm{HPO}_{4}$ and the other ingredients described above except MOPS is referred to as control medium. When an increase in $\mathrm{NH}_{4}{ }^{+}$or $\mathrm{PO}_{4}{ }^{3-}$ content of the defined medium was required, $\left(\mathrm{NH}_{4}\right)_{2} \mathrm{SO}_{4}$ and $\mathrm{K}_{2} \mathrm{HPO}_{4}$ solutions were added.

$\left[{ }^{14} \mathrm{C}\right]$ Protylonolide Production from ${ }^{14} \mathrm{C}$-Labeled Compounds with Washed Mycelia of Strain 261

Strain 261 was grown in test tubes at $27^{\circ} \mathrm{C}$. At daily intervals, the mycelia were harvested by centrifugation $\left(5,000 \mathrm{rpm}, 10\right.$ minutes at $\left.5^{\circ} \mathrm{C}\right)$, then washed twice with and suspended in an equal volume of $0.1 \mathrm{M}$ MOPS ( $\mathrm{pH} 7.8$ ). To a $2-\mathrm{ml}$ aliquot of this suspension (6 8 $\mathrm{mg}$ dry mycelial weight/ $\mathrm{ml}$ ) in a test tube $(1.7 \times 10 \mathrm{~cm})$ were added $20 \mu \mathrm{l}$ of ${ }^{14} \mathrm{C}$-labeled compound (final concentration of $0.2 \mu \mathrm{Ci} /$ $\mathrm{ml}$ ) and $20 \mu \mathrm{l}$ of the corresponding unlabeled compound (final concentration of $2 \mathrm{~mm}$ ). The reaction mixture was incubated at $27^{\circ} \mathrm{C}$ with reciprocal shaking (120 strokes/minute). After 5-hour shaking, the reaction mixture was centrifuged $\left(3,000 \mathrm{rpm}, 10\right.$ minutes at $\left.5^{\circ} \mathrm{C}\right)$ and the supernatant fluid was used for $\left[{ }^{14} \mathrm{C}\right]$ protylonolide assay. Under the conditions specified above, $\left[{ }^{14} \mathrm{C}\right]$ protylonolide production increased linearly up to 6 hours. Without supplement of unlabeled compounds, protylonolide production was sigmoidal and reached a plateau within two to three hours.

\section{Analytical Methods}

Dry cell weight and the amount of unlabeled protylonolide were determined as described previously. ${ }^{1)}$ The protylonolide titer reported here is actually the total amount of protylonolide and the co-produced shunt metabolite mycarosylprotylonolide.

For the radioassay of ${ }^{14} \mathrm{C}$-labeled protylonolide, reaction mixtures in three test tubes incubated under identical reaction conditions were combined and centrifuged. Supernatant fluid $(0.5 \mathrm{ml})$ was extracted once with $1.5 \mathrm{ml}$ of toluene. The toluene layer $(1.0 \mathrm{ml})$ was washed twice with an equal volume of $0.01 \%$ aqueous formic acid solution. The washed toluene layer $(0.5 \mathrm{ml})$ was evaporated to dryness and the residue, mixed with $5 \mathrm{ml}$ of toluene-based scintillation cocktail (Amersham, England), was assayed for radioactivity with an Aloka liquid scintillation spectrometer (model LSC-653).

\section{Autoradiography}

Two $\mathrm{ml}$ of the washed toluene layer obtained as above were concentrated to dryness, dissolved in $0.1 \mathrm{ml} \mathrm{MeOH}$, and applied to a silica gel thin-layer plate $(0.2 \mathrm{~mm}$, E. Merck, Art $\# 5554)$, which was developed with $\mathrm{CHCl}_{3}-\mathrm{MeOH}(20: 1)$ as solvent. After drying, the silica gel layer was placed on a Kodak X-ray film (XR-1) and left in the dark for a month at room temperature. The film was then developed.

\section{Chemicals}

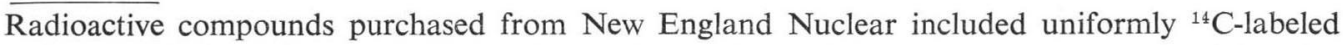
valine, leucine, isoleucine, threonine, alanine, lysine and palmitic acid, and $\left[2,3-{ }^{14} \mathrm{C}_{2}\right]$ succinic acid. Other chemicals were obtained from commercial sources and were of reagent grade, except for media ingredients. 


\section{Results}

Inhibition of Protylonolide Production by Ammonium Ions

in a Chemically Defined Medium

A previous paper showed that $\mathrm{NH}_{4}{ }^{+}$or its metabolic analog methylamine inhibited protylonolide production in a complex medium. ${ }^{1)}$ The effect was re-examined in a chemically defined medium (control medium) which contained $25 \mathrm{mM} \mathrm{NH}_{4}{ }^{+}$and $2.9 \mathrm{mM} \mathrm{PO}_{4}{ }^{3-}$. The addition of $50 \mathrm{mM} \mathrm{NH}_{4}{ }^{+}$ or $10 \mathrm{mM} \mathrm{PO}_{4}{ }^{3-}$ to the control medium decreased specific protylonolide production (i.e. $\mu \mathrm{g}$ of protylonolide producted per mg of dry mycelial weight) by 73 or $79 \%$, respectively, at day 4 . Ammonium sulfate and ammonium chloride had similar effects.

The above inhibition was accompanied by small changes in $\mathrm{pH}$ values from those of the control

Fig. 1. Effect of ammonium ions on growth of $S$. fradiae strain 261 and protylonolide production in a defined medium under $\mathrm{pH}$-control.

Strain 261 was grown at $27^{\circ} \mathrm{C}$ in test tubes containing $10 \mathrm{ml}$ of MOPS-buffered defined media. The $\mathrm{NH}_{4}{ }^{+}$concentrations of the defined media were $25(\bigcirc), 50(\triangle), 75(\bullet)$ and $100(\square) \mathrm{mm}$, as indicated. Mean values of triplicate tubes for growth and $\mathrm{pH}$ (upper half) and protylonolide titers (lower half) are shown.
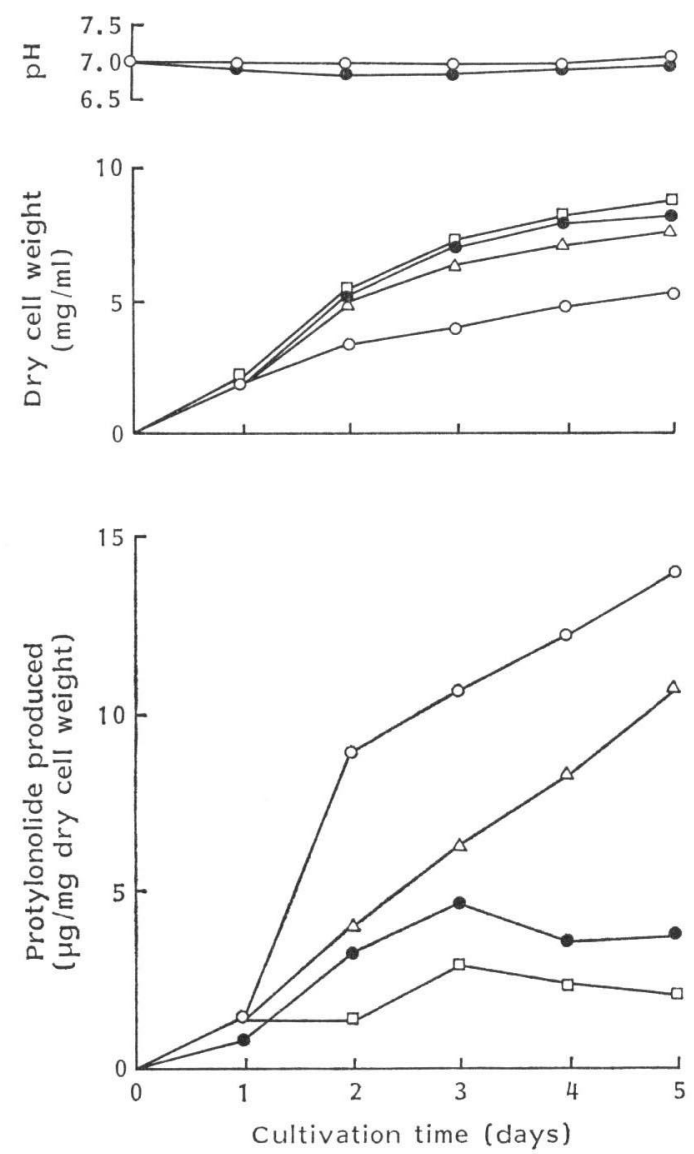
medium with no additions. To avoid possible pH effects, the medium was supplemented with the Goop buffer, MOPS. Varying amounts of ammonium sulfate were then added to deter-

Fig. 2. Inhibition of protylonolide production by $\mathrm{NH}_{4}{ }^{+}$and its reversal by lower fatty acids.

$S$. fradiae strain 261 was grown for 5 days in control medium except that $50 \mathrm{mM} \mathrm{NH}_{4}{ }^{+}$was contained initially. At day 1 , the test tubes received solutions $(0.1 \mathrm{ml})$ of either one or both of the indicated lower fatty acids $(\mathrm{pH} 7)$ and $\left(\mathrm{NH}_{4}\right)_{2} \mathrm{SO}_{4}$ at the given final concentrations, and incubated for a further 4 days. Protylonolide titers per mg dry cells at day 5 are shown.

$\mathrm{a}$ : No addition, b: $n$-butyric acid $(n$-BA) + propionic acid (PrA) + acetic acid (AcA) $(0.2 \mathrm{mg} / \mathrm{ml})$, c: no addition, d: $i$-butyric acid $(0.5 \mathrm{mg} / \mathrm{ml})$, e: $n$-butyric acid $(0.5 \mathrm{mg} / \mathrm{ml}), \mathrm{f}:$ 2-ketoisovaleric"acid $+i$-butyric acid $(0.5 \mathrm{mg} / \mathrm{ml})$.

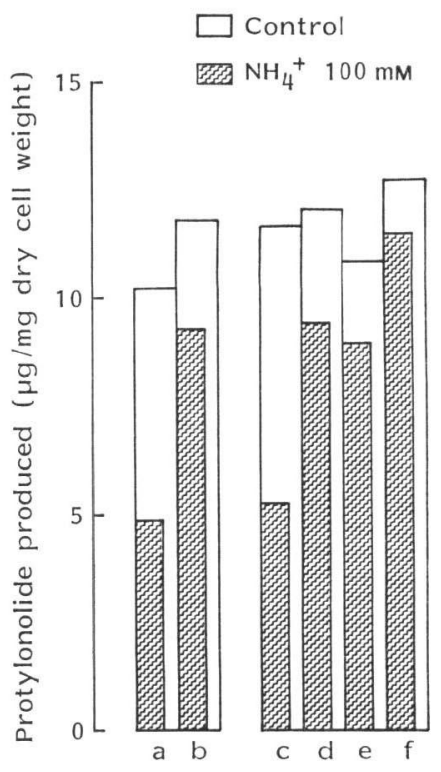


mine the effects on growth and protylonolide production. Under such conditions, the ammonium salt increased growth of the protylonolide-producing mutant and caused only a small decrease of $\mathrm{pH}$ values (Fig. 1). The increases in the amount of ammonium supplement resulted in a steep reduction in specific titer of protylonolide.

\section{Reversal by Lower Fatty Acids of the Inhibitory Effect of Ammonium Ions on Protylonolide Production}

In one approach to study the site of ammonium action, compounds which reversed the inhibitory effect of $\mathrm{NH}_{4}{ }^{+}$were examined. To one-day cultures which contained about $20 \mathrm{~mm}$ of the initial $50 \mathrm{mM} \mathrm{NH}_{4}{ }^{+}$were added various compounds either alone or mixed with $100 \mathrm{mM} \mathrm{NH}_{4}{ }^{+}$. In a culture receiving only $100 \mathrm{mM} \mathrm{NH}_{4}{ }^{+}$, protylonolide production was about $50 \%$ of the control titer at day 5 . Fig. 2 illustrates that the simultaneous addition of $n$-butyrate, propionate and acetate restored protylonolide production. Isobutyrate alone or in combination with 2-ketoisovalerate was as effective as the above three lower fatty acids. This is noteworthy, because both acids are products of valine catabolism. Although addition of lower fatty acids alone to the control culture somewhat increased protylonolide titers, nevertheless $80 \sim 90 \%$ restoration was observed. These results suggest that the site of action of $\mathrm{NH}_{4}{ }^{+}$lies within steps preceding the formation of lower fatty acid precursors. The medium used in the above reversal experiment was different from the control medium employed in the experiment of Fig. 1. Later, sufficient reversal was also observed in similar experiments using 500-ml conical flasks containing MOPS-buffered control medium supplemented with $50 \mathrm{mM} \mathrm{NH}_{4}{ }^{+}$and lower fatty acids.

\section{Short-term Production of Protylonolide by Washed Mycelia with ${ }^{14} \mathrm{C}$-Labeled Compounds as Substrates}

Lower fatty acids are produced in the metabolism of amino acids and of higher fatty acids, as well as in the tricarboxylic acid (TCA) cycle. These metabolic pathways are therefore potential sources of precursors for protylonolide biosynthesis. The results described above suggest that regulation by $\mathrm{NH}_{4}{ }^{+}$operates in these pathways. In order to study which route is preferred for protylonolide biosynthesis and is relevant to regulation by $\mathrm{NH}_{4}{ }^{+}$, we developed a resting cell system in which shortterm production of protylonolide with potential sources of precursors as substrates was monitored by a sensitive radioisotope assay.

Preliminary experiments showed that washed mycelia of the protylonolide producer S. fradiae strain 261 accumulated $\left[{ }^{14} \mathrm{C}\right]$ protylonolide when incubated in a buffer system with $\left[{ }^{14} \mathrm{C}\right]$ valine plus $2 \mathrm{~mm}$ cold valine. The optimal $\mathrm{pH}$ for protylonolide production was about 8 with valine as substrate and with MOPS as buffering agent. MOPS was the best among the buffers tested, which included other Good buffers and potassium phosphate, within a range of $\mathrm{pH} 6 \sim 10$. Under the conditions used, protylonolide and mycarosylprotylonolide were the sole radioactive products revealed by autoradiography (not shown). Specific radioactivities of products from labeled valine were nearly equal regardless of $\mathrm{NH}_{4}{ }^{+}$levels of media in which mycelia were previously grown. A different specific value was obtained with labeled succinate as the substrate.

Table 1 (expt 1) shows that $\left[U-{ }^{14} \mathrm{C}\right]$ valine gave a higher radioactive yield in protylonolide than $\left[2,3-{ }^{14} \mathrm{C}_{2}\right]$ succinate (a compound of the TCA cycle), $\left[U-{ }^{14} \mathrm{C}\right]$ palmitate (a higher fatty acid) or $\left[U-{ }^{14} \mathrm{C}\right]-$ glucose (a general source of lower fatty acids). In a previous paper, ${ }^{2)}$ several amino acids were shown to promote protylonolide production in a growing culture of strain 261 . These amino acids were 
Table 1. Production of $\left[{ }^{14} \mathrm{C}\right]$ protylonolide by resting cells of $S$. fradiae strain $261 .{ }^{\text {a }}$

\begin{tabular}{lc|cc}
\hline $\left.\begin{array}{c}\text { Addition } \\
0.2 \mu \mathrm{Ci} / \mathrm{ml} \\
2 \mathrm{~mm}\end{array}\right)$ & $\begin{array}{c}{ }^{14} \mathrm{C} \text { Protylonolide } \\
\text { produced } \\
(\%)\end{array}$ & $\left.\begin{array}{c}\text { Addition } \\
0.2 \mu \mathrm{Ci} / \mathrm{ml} \\
2 \mathrm{mM}\end{array}\right)$ & $\begin{array}{c}\left.{ }^{14} \mathrm{C}\right] \text { Protylonolide } \\
\text { produced }\end{array}$ \\
$($ Expt 1$)$ & $100^{\mathrm{b})}$ & Valine & 1,300 \\
Valine & 32 & Threonine & 1,000 \\
Succinic acid & $39^{\mathrm{c})}$ & Isoleucine & 400 \\
Palmitic acid & 18 & Leucine & 400 \\
Glucose & & Alanine & 300 \\
& & Lysine & 50 \\
\hline
\end{tabular}

a) S. fradiae strain 261 was grown as in Fig. 1, but without pH-control. At day 2 cells were harvested and washed, and incubated with the indicated labeled compound in $0.1 \mathrm{M}$ MOPS (pH 7.8 ) at $27^{\circ} \mathrm{C}$ for 5 hours. The radioactivity of the protylonolide fraction was counted.

b) $100 \%=15,600 \mathrm{dpm} / \mathrm{ml}$. Ca. $8 \mathrm{mg} / \mathrm{ml}$ (not determined exactly) of mycelia was used.

c) The reaction tube was supplemented with $1 \mu \mathrm{g} / \mathrm{ml}$ of the surfactant Adekanol LG-109 (Asahi Denka, Tokyo). In the radioassay, the toluene layer was thoroughly washed.

used as substrates for protylonolide production by washed mycelia. Table 1 (expt 2) indicates that valine gave the highest radioactive yield in protylonolide, followed by threonine, isoleucine, leucine and alanine. Lysine, which did not promote protylonolide formation in growing cultures, was least incorporated.

These results show that not only valine but other amino acids in addition to succinic and palmitic acids can serve as sources of building units for protylonolide biosynthesis. Valine was the best among the compounds tested.

Suppression by Ammonium Ions of Labeled Protylonolide

Production by Washed Mycelia

The effect of ammonium sulfate on the incorporation of amino acids into protylonolide was studied using washed mycelia. S. fradiae strain 261 was grown in the same manner as in Fig. 1 in defined medium supplemented with varying amounts of ammonium sulfate and with $0.1 \mathrm{M}$ MOPS. The mycelia, harvested and washed every day, were used for the production of labeled protylonolide from $\left[{ }^{14} \mathrm{C}\right]$ amino acids.

Fig. 3 (left half) shows daily changes in protylonolide productivity with $\left[{ }^{14} \mathrm{C}\right]$ valine as substrate and with mycelia previously grown in the presence of two levels of ammonium sulfate. The control mycelia (grown with $25 \mathrm{mM} \mathrm{NH}_{4}{ }^{+}$) showed the highest productivity at day 1, at which time protylonolide accumulation had just begun in a growing culture (see Fig. 1). When the concentration of ammonium salt increased in the growth medium, protylonolide productivity fell steeply. Washed mycelia from cultures grown in the presence of $100 \mathrm{mM} \mathrm{NH}_{4}{ }^{+}$produced almost no protylonolide.

With $\left[{ }^{14} \mathrm{C}\right]$ threonine as starting material, the protylonolide-producing activity of the control mycelia peaked at day 2 instead of at day 1 as seen with valine as substrate. Cells previously grown on higher amounts of $\mathrm{NH}_{4}{ }^{+}$incorporated less label. With leucine, isoleucine or alanine as substrate, the control mycelia exhibited lower productivity than with valine or threonine throughout the period studied. Mycelia grown under $\mathrm{NH}_{4}{ }^{+}$-rich conditions showed lower activity than the control (data not shown). Lysine did not substantially support protylonolide production irrespective of the time of mycelial harvest. 
Fig. 3. Incorporation of ${ }^{14} \mathrm{C}$-labeled amino acids into protylonolide by washed cells of $S$. fradiae strain 261 previously grown in low and high levels of ammonium ions.

Strain 261 was grown in 25, 50, 75 and $100 \mathrm{mM} \mathrm{NH}_{4}{ }^{+}$under $\mathrm{pH}$-control as in Fig. 1. Cells were harvested and washed daily, and then incubated with the indicated amino acids $(2 \mu \mathrm{Ci} / \mathrm{ml}, 2 \mathrm{~mm})$ in MOPS buffer as described in Table 1. Mean radioactivity of protylonolide produced in triplicate reaction tubes is shown. The results with cells grown in 50 and $100 \mathrm{~mm} \mathrm{NH}_{4}{ }^{+}$were omitted here for simplicity.

$\mathrm{NH}_{4}+25 \mathrm{~mm}, \mathrm{NH}_{4}+75 \mathrm{~mm}$.

$\left[{ }^{14} \mathrm{C}\right]$ Valine

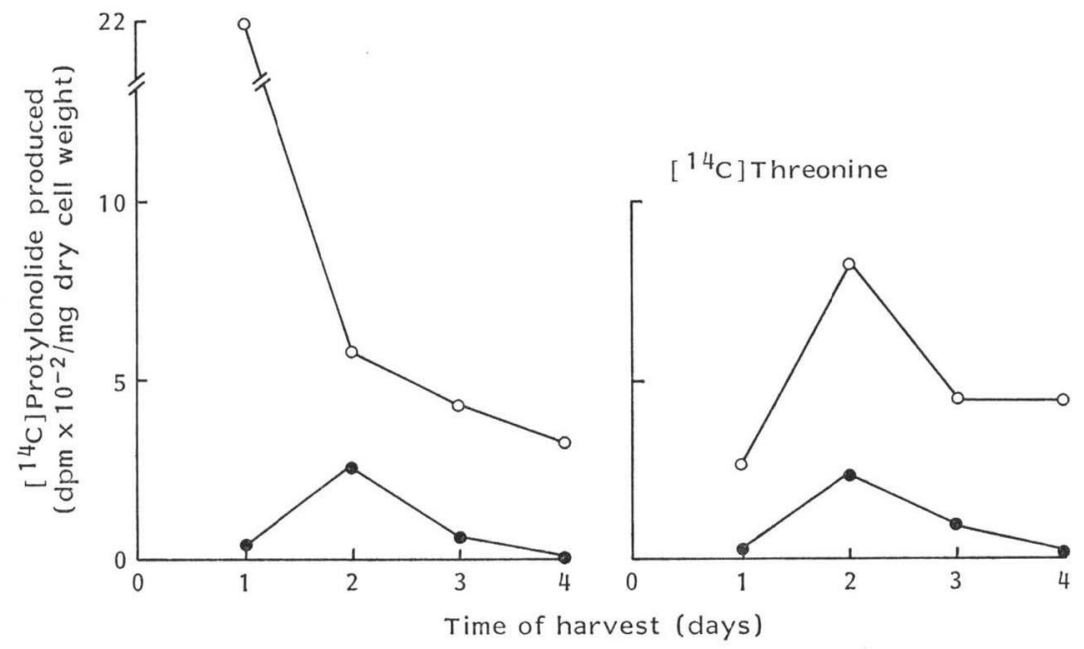

Discussion

Amino acids are metabolized to produce lower fatty acids in various microorganisms. Because protylonolide arises from only $n$-butyrate, propionate and acetate units as direct precursors, the efficient incorporation of several amino acids into protylonolide (Table 1) is a strong indication that they were utilized as sources of lower fatty acid precursors. A similar result was recently reported by DotzLAF et $a l .^{\left.{ }^{8}\right)}$ who also found that lysine was poorly incorporated even though they studied under different conditions.

The ratio of the label incorporated into protylonolide was 100:32: 39 with valine, succinate and palmitate as substrates (Table 1, expt 1). This is suggestive of an important role of amino acid metabolism in protylonolide biosynthesis. ŌMURA et al. ${ }^{5)}$ showed that one $n$ butyrate, five propionate and two acetate units participate in the biosynthesis of tylosin aglycone. They also demonstrated that valine was metabolized to $n$-butyrate in addition to the normally expected propionate unit used for protylonolide biosynthesis in $S$. fradiae strain 261.8) Under conditions which favor catabolism, n-butyrate

Table 2. Lower fatty acids produced by normal metabolism and number of protylonolide carbons possibly labeled by ${ }^{14} \mathrm{C}$-labeled amino acid- and organic acid-derived lower fatty acids.

\begin{tabular}{llc}
\hline \multicolumn{1}{c}{ Source $^{\mathrm{a})}$} & $\begin{array}{c}\text { Lower fatty acids } \\
\text { produced }\end{array}$ & $\begin{array}{c}\text { Carbons } \\
\text { labeled }\end{array}$ \\
\hline Valine & AcA, PrA, $n-\mathrm{BA}^{\mathrm{c})}$ & 23 \\
Threonine & PrA & 15 \\
Isoleucine & PrA & 15 \\
Leucine & $\mathrm{AcA}, \mathrm{AAA}^{\mathrm{d})}$ & 8 \\
Alanine & $\mathrm{AcA}$ & 4 \\
{$\left[2,3-{ }^{14} \mathrm{C}_{2}\right]-$} & & \\
$\quad$ Succinic acid & PrA $^{\mathrm{e})}$ & 10 \\
Palmitic acid & $\mathrm{AcA}, n-\mathrm{BA}$ & 8 \\
\hline
\end{tabular}

a) Unless otherwise specified, compounds are uniformly ${ }^{14} \mathrm{C}$-labeled.

b) Abbreviations are the same as in Fig. 2. CoA esters and $\mathrm{CoA}$ esters of 2-carboxylated derivatives of these acids are biosynthetically equivalent, and are not discriminated.

c) From ŌMura et $a l{ }^{8)}$

d) AAA, acetoacetic acid; from DotzlaF et al..$^{9)}$

e) From ŌMURA et al. ${ }^{\text {) }}$ and Dotzlaf et al. ${ }^{9)}$ 
readily produces acetate, but not vice versa. Taking these facts into consideration, together with the generally accepted scheme of amino acid metabolism, the lower fatty acids derived from ${ }^{14} \mathrm{C}$ labeled amino acids and from organic acids are expected to label the 23 carbons of protylonolide as shown in Table 2.

The ratio of label by valine and palmitate (100:39) (Table 1, expt 1) was somewhat higher than the expected value of $23: 8$ (Table 2). On the other hand, the ratio of valine to succinate (100:32) was a little lower than the calculated value, 23:10. The gap in the latter case is ascribed to lower production of protylonolide with succinate as substrate because of limited availability of acetate and/or $n$-butyrate precursor units, or to low permeability of succinate. Notwithstanding, it is clear that valine was as good a source of precursor as succinate and palmitate. The role of other amino acids can be assessed on the same basis.

Previous observation indicated that care has to be taken, however, in the above interpretation. Firstly, amino acid metabolism in $S$. fradiae does not always follow the general routes. ${ }^{8)}$ Secondly, because interconversion of $n$-butyrate, propionate and acetate units is involved as minor metabolism in tylosin biosynthesis, ${ }^{6,7,10)}$ amino acid normally expected to serve as sources of, for example, propionate can also supply small fractions of acetate and/or $n$-butyrate units. In addition, the amino acids actually participating in protylonolide biosynthesis vary with culture age (compare valine and threonine in Fig. 3), culture conditions, and other metabolic activities. It might be that valine and threonine were preferred under the particular conditions employed here. Further studies are required to determine the labeling pattern of individual amino acids.

The incorporation of radioactivity from all the amino acids tested, except lysine, into protylonolide markedly decreased when the cells were previously grown in high $\mathrm{NH}_{4}{ }^{+}$conditions (Fig. 3). This result, together with the results shown in Figs. 1 and 2, can be reasonably explained as suppression by $\mathrm{NH}_{4}{ }^{+}$of amino acid metabolism to lower fatty acids. The mechanism by which $\mathrm{NH}_{4}{ }^{+}$causes such a pleiotropic effect on amino acid metabolism is not known. We could not assess the inhibitory effect of $\mathrm{NH}_{4}{ }^{+}$on the activity of amino acid-metabolizing enzymes because $\mathrm{NH}_{4}{ }^{+}$added to the resting cell system also affected amino acid transport (not shown).

The severe reduction of amino acid incorporation by $\mathrm{NH}_{4}{ }^{+}$can arise from two possible patterns of regulation: (1) The pathways starting from amino acids, succinate and higher fatty acids are all suppressed by $\mathrm{NH}_{4}{ }^{+}$, or (2) the major route starting from one or two groups of compounds (amino acids, for instance) is sensitive to the $\mathrm{NH}_{4}{ }^{+}$effect, but the rest are not. To examine these possibilities, studies are now in progress on the effect of $\mathrm{NH}_{4}{ }^{+}$on succinate and palmitate metabloism.

The results presented in this paper provide a practical basis for a study aimed at higher production of tylosin and related macrolide antibiotics by increasing both the biosynthesis and the degradation of relevant amino acids. The enzymatic bases of the present results will be described in a forthcoming paper.

\section{References}

1) Masuma, R.; Y. TANaka \& S. ŌMura: Ammonium ion-depressed fermentation of tylosin by the use of a natural zeolite and its significance in the study on the biosynthetic regulation of the antibiotic. J. Ferment. Technol. 61: 607 614, 1983

2) Ōmura, S.; Y. TAnaKa, H. Mamada \& R. Masuma: Effect of ammonium ion, inorganic phosphate and amino acids on the biosynthesis of protylonolide, a precursor of tylosin aglycone. J. Antibiotics 37 : $494 \sim 502,1984$

3) Baltz, R. H. \& E. T. Seno: Properties of Streptomyces fradiae mutants blocked in biosynthesis of the macrolide antibiotic tylosin. Antimicrob. Agents Chemother. 20: 215 225, 1981

4) Ōmura, S.; C. KitAo \& H. Matsubara: Isolation and characterization of a new 16-membered lactone, protylonolide, from a mutant of tylosin-producing strain, Streptomyces fradiae KA-427. Chem. Pharm. Bull. 29: $1963 \sim 1965,1980$

5) Ōmura, S.; A. Nakagawa, H. Takeshima, J. Miyazawa, C. Kitao, F. Piriou \& G. Lukacs: A ${ }^{13}$ C nuclear magnetic resonance study of the biosynthesis of the 16-membered macrolide antibiotic tylosin. Tetrahedron Lett. 1975: 4503 4506, 1975 
6) Ōmura, S.; H. Takeshima, A. Nakagawa, N. Kanemoto \& G. Lukacs: Studies on carboxylic acid metabolism in a macrolide-producing microorganism using carbon-13 magnetic resonance. Bioorg. Chem. 5: $451 \sim 454,1976$

7) Ōmura, S.; H. Takeshima, A. Nakagawa, J. Miyazawa, F. Piriou \& G. Lukacs: Studies on the biosynthesis of 16-membered macrolide antibiotics using carbon-13 nuclear magnetic resonance spectroscopy. Biochemistry 16: 2860 2866, 1977

8) Ōmura, S.; K. Tsuzuki, Y. Tanaka, H. Sakakibara, M. Aizawa \& G. Lukacs: Valine as a precursor of $n$-butyrate unit in the biosynthesis of macrolide aglycone. J. Antibiotics 36: 614 616, 1983

9) Dotzlaf, J. E.; L. S. Metzger \& M. A. Foglesong: Incorporation of amino acid-derived carbon into tylactone by Streptomyces fradiae GS 14. Antimicrob. Agents Chemother. 25: 216 220, 1984

10) O'Hagen, D.; J. A. Robinson \& D. L. Turner: Biosynthesis of the macrolide antibiotic tylosin. Origin of the oxygen atoms in tylactone. J. Chem. Soc., Chem. Commun. 1983: 1337 1340, 1983 